
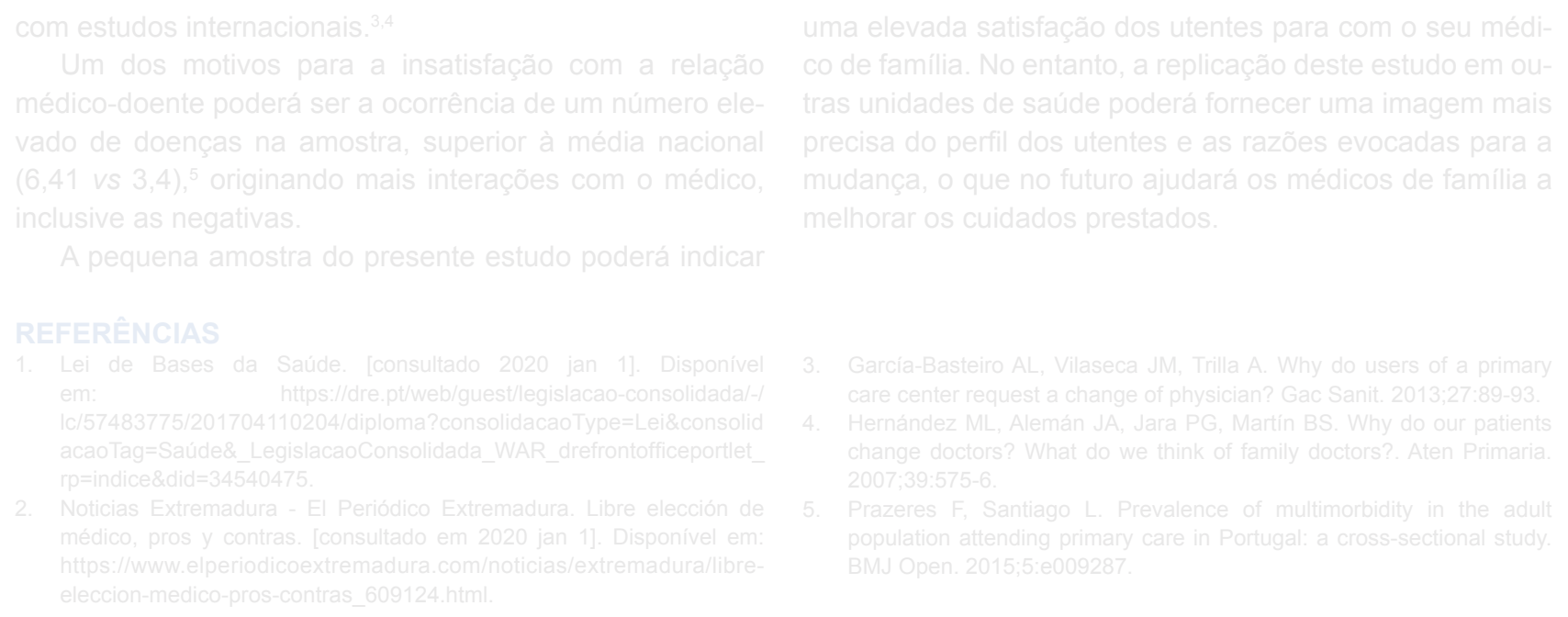

\section{Alterações Neuropsicológicas e Indicação de Cirurgia Bariátrica em Doentes Obesos Graves}

Neuropsychological Changes and Indication of Bariatric Surgery in Severely Obese Patients

Palavras-chave: Cognição; Emoções; Funções Executivas; Obesidade Mórbida/psicologia; Portugal; Reserva Cognitiva

Keywords: Cognition; Cognitive Reserve; Emotions; Executive Function; Obesity, Morbid/psychology; Portugal

Muito importante o artigo abordando o perfil neuropsicológico, reserva cognitiva e desajustamento emocional numa amostra portuguesa de doentes com obesidade grave. ${ }^{1} \mathrm{~A}$ obesidade é a maior epidemia do século XXI e está associada a uma gama de comorbidades graves que vão desde hipertensão arterial, diversos tipos de neoplasias malignas e alterações neuropsicológicas. As alterações psicológicas e neurológicas nesses pacientes impõem ainda mais a necessidade de indicação de um tratamento para a obesidade para diminuir o número de comorbidades associadas.

Todavia, por se tratar de um estudo transversal no qual se utilizou um questionário sociodemográfico e clínico, provas neuropsicológicas e de avaliação de sintomas de desajustamento emocional, na avaliação do desempenho cognitivo de pacientes, não temos como estabelecer relação de causa-efeito, isto é, se a obesidade foi consequência das alterações neurológicas ou se ela foi a causa das mesmas. Sendo assim, acreditamos que não devemos retardar o tratamento adequado para o tratamento da obesidade, principalmente a indicação de cirurgia bariátrica nesses pacientes portadores de obesidade grave ${ }^{2}$. As intervenções de mudanças do estilo de vida, terapia cognitivo comportamental, grupos de apoio e treino cognitivo são importantes para uma abordagem complementar do paciente, mas não devem retardar ou atrasar a indicação de cirurgia bariátrica.

Existem estudos que avaliaram os efeitos da gastrectomia em manga ou sleeve e bypass gástrico na função cognitiva e na associação entre perda de peso e controle da compulsão alimentar e mostraram melhora com a cirurgia bariátrica. $^{3}$

A cirurgia bariátrica não se centra diretamente no tratamento de alguma alteração comportamental ou psicológica subjacente que esteja a contribuir para a obesidade, mas é o tratamento 'padrão-ouro' para o tratamento da obesidade grave. ${ }^{2,4}$ Acreditamos, bem como outros autores, que a sua indicação não deva ser retardada nesse grupo de pacientes. ${ }^{4}$ Nunca esquecendo que o sucesso da cirurgia bariátrica não se mede apenas pela perda de peso, mas também pela melhoria ou cura das comorbilidades clínicas associadas. É fundamental manter um adequado acompanhamento pós-operatório após a cirurgia com equipe multidisciplinar, e quando indicado, o tratamento psiquiátrico deve ser instituído o mais rapidamente possível. ${ }^{5}$ 


\section{REFERÊNCIAS}

1. Ribeiro O, Carmo I, Paiva T, Figueira ML. Neuropsychological profile, cognitive reserve and emotional distress in a Portuguese sample of severely obese patients. Acta Med Port. 2020;33:38-48.

2. Chang SH, Stoll CR, Song J, Varela JE, Eagon CJ, Colditz GA. The effectiveness and risks of bariatric surgery: an updated systematic review and meta-analysis, 2003-2012. JAMA Surg. 2014;149:275-87.

3. Pepino MY, Stein RI, Eagon JC, Klein S. Bariatric surgery-induced weight loss causes remission of food addiction in extreme obesity.
Obesity. 2014;22:1792-8.

4. Cooiman MI, Aarts EO, Janssen IM, Hazebroek EJ, Berends FJ. Weight loss, remission of comorbidities, and quality of life after bariatric surgery in young adult patients. Obes Surg. 2019;29:1851-7.

5. Gordon KH, King WC, White GE, Belle SH, Courcoulas AP, Ebel FE, et al. A longitudinal examination of suicide-related thoughts and behaviors among bariatric surgery patients. Surg Obes Relat Dis. 2019;15:269-78.

\section{Eduardo Neubarth TRINDADE $\rrbracket^{1}$, Manoel Roberto Maciel TRINDADE ${ }^{1,2}$}

1. Serviço de Cirurgia Digestiva. Programa de Cirurgia Bariátrica. Hospital de Clínicas de Porto Alegre. Porto Alegre. Brasil.

2. Departamento de Cirurgia. Universidade Federal do Rio Grande do Sul. Rio Grande do Sul. Brasil.

Autor correspondente: Eduardo Neubarth Trindade. eduardontrindade@yahoo.com.br

Recebido: 06 de janeiro de 2020 - Aceite: 08 de janeiro de 2020 | Copyright $\odot$ Ordem dos Médicos 2020

https://doi.org/10.20344/amp.13385 